\title{
AUTONOMOUS MACHINING SYSTEMS
}

\author{
Tran Ngoc Hien \\ Faculty of Mechanical Engineering, University of Transport and Communications, No.3 Cau \\ Giay Street, Lang Thuong Ward, Dong Da District, Hanoi, Vietnam \\ Email: ulsanuni@gmail.com
}

Received: 20 August 2016; Accepted for publication: 20 February 2017

\begin{abstract}
Intelligent machining systems enable to adapt to the changes of manufacturing environment such as orders, disturbances happening in the system. In machining system, machine tools play an important role to have the products with high quality, low cost and high productivity. The paper presents a new concept of intelligent machining system, namely Autonomous Machining System- AMS. Biology inspired technologies are applied to the machine tools for equipping these machines with biological characteristics such as self-diagnostic, self-recovery, and cooperation. With these advanced characteristics, AMS has ability to adapt to changes on the shop floor.
\end{abstract}

Keywords: autonomous machining system, artificial intelligence, cognitive agent.

\section{INTRODUCTION}

In traditional manufacturing systems, the workers play an important role, with their knowledge and experience, the workers adapt flexibly to changes in the manufacturing environment. With new problems, their knowledge is updated through learning [1]. With abilities in processing information and cognitive abilities, the workers keep an important role in monitoring, control, production planning. The workers with the ability to solve problems and cognitive capacity enable to adapt with changing manufacturing environment. However, the system manipulated by workers with a high price is only suitable for small production.

In the era of computer integrated manufacturing, workers are replaced by automatic control systems and robots, so the cognitive abilities of workers in solving problems such as perception, learning, reasoning for a decision also are removed [2]. The limit of the automatic control system is not capable to adapt to the changes due to the system operating under preset programs. So the system needs to reset and restart when an error occurs. To overcome these shortcomings, and combination of both advanced automatic control system and the cognitive abilities of human, cognitive sciences and artificial intelligence as well as the biology inspired technologies have been applied to the manufacturing systems which make the production system to become more intelligent and more flexible [3 - 5]. Kristina et al. proposed a manufacturing system with 
applying the achievements of cognitive science. In this system, the machines work together for machining workpiece. The system adapts flexibly with the change of orders and changes in the machining shop [6].

The paper presents the application of biology inspired technologies allowing the system to perform intelligent behaviors which mimic human activities such as reasoning for decisionmaking, cooperation in problem solving.

\section{MODEL OF AN AUTONOMOUS MACHINING SYSTEM}

With the important role of machine tools in machining systems, many research projects have proposed technological solutions to get the intelligent machine tools such as applying the adaptive control [7], intelligent controller [8], re-configuration of the machine tools [9], or online system for monitoring processes [10] which allow the machine to adapt to the changes in the production environment, ensuresthe product quality. Control solutions to improve the flexibility and extend the workspace of the machine have also been studied [11].

The concept of an autonomous machine tool (AMT) was first mentioned in the 1980s [12 14]. Studies on AMT have focused on themachining process model of the intelligent machine tools, multi-sensor networks, identification and fault diagnosis. An AMS has many AMTs which cooperate to perform the machining process.

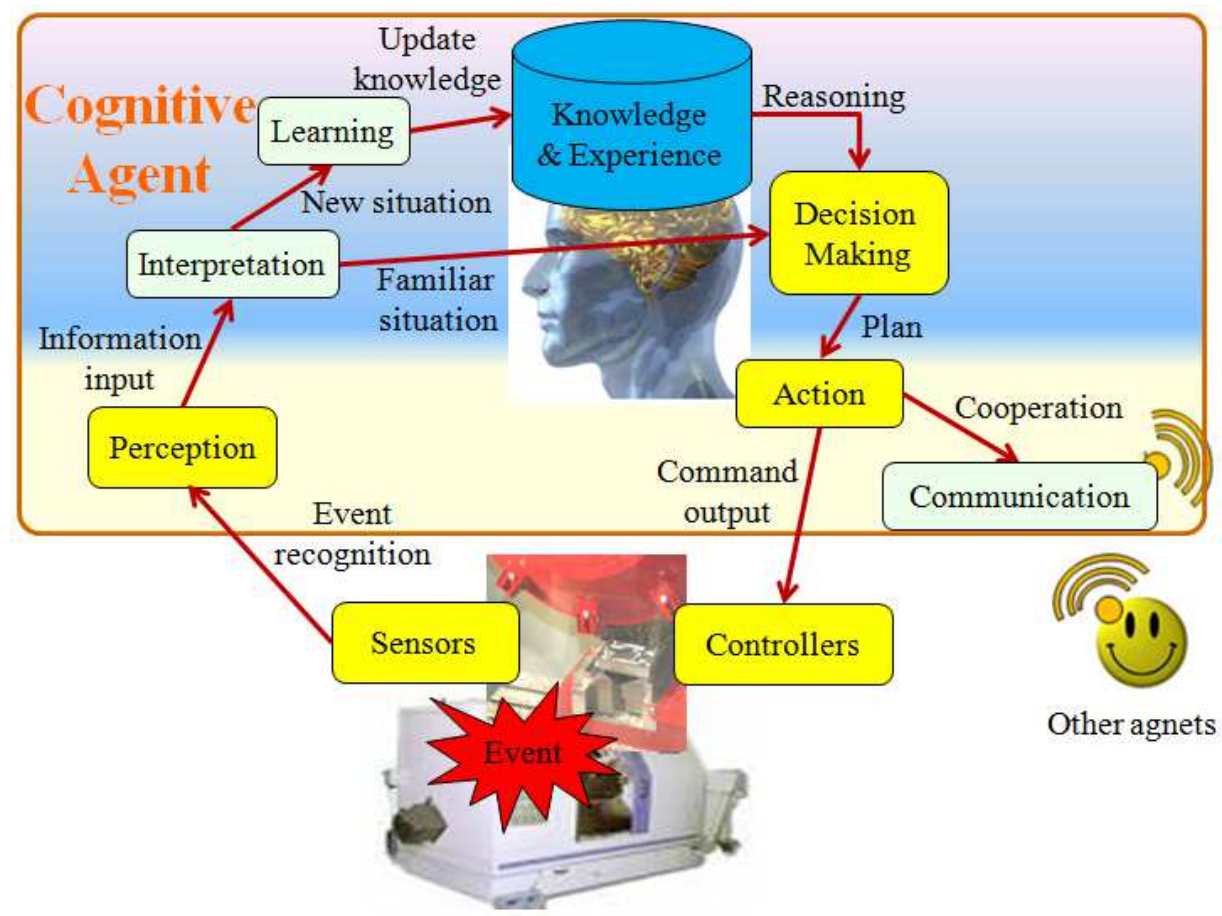

Figure 1.Model of an autonomous machine tool in AMS.

Currently, self-adapting thecuttingconditions in machining process on the machine tool was made successfully by applying adaptive controller. The adaptive control systems for machine tools are classified into three groups: adaptive control with constraints (ACC), geometric 
adaptive control (GAC), and adaptive control with optimization (ACO) [15, 16]. ACC is based on the maximum value of processing variables such as cutting speed in cosideration of the machine and cutting process such as cutting force, and machine power. With the GAC, the cutting process parameters are continuously adjusted in relation to the geometric precision and workpiece surface quality. ACO adjusts the machining process parameters such as feed rate, cutting speed and depth of cut to get the largest amount of material removed with the constraints of the surface roughness, the power consumption and cutting force. Virtual reality technology has also been proposed to apply for the CNC milling machine, in which the digital copy milling system is connected to the CNC machine, the change of cutting conditions or tool paths on the digital system will be updated on a real machining system [17].

The paper presents the research results on AMS with the application of cognitive agent technology as shown in Figure 1. Cognitive agents integrated from agent technology and cognitive technology is a computer program which are equipped with artificial cognitive abilities to perform cognitive activities from the behavior of human such as perception, reasoning and decision-making, communication, and learning $[18,19]$. The intelligent features of the machine tools are carried out by cognitive agents. Difference with the adaptive control system mentioned above, this research considers to self-adapting to disturbances of each machines as well as of the systemdue to cooperation among machines in order to reduce the downtime of the machine or the system in the case of an error happening such as tool wear, machine breakdown when machining product.

The model of an autonomous manufacturing system (AMS) is shown in Figure 2. Each machine tool is equipped with artificial cognitive abilities so that it can perform intelligent behavior such as perception, decision making, and communication. For machining a new product, manufacturing execution system (MES) sends the plans to the machine tools via the cognitive agents.

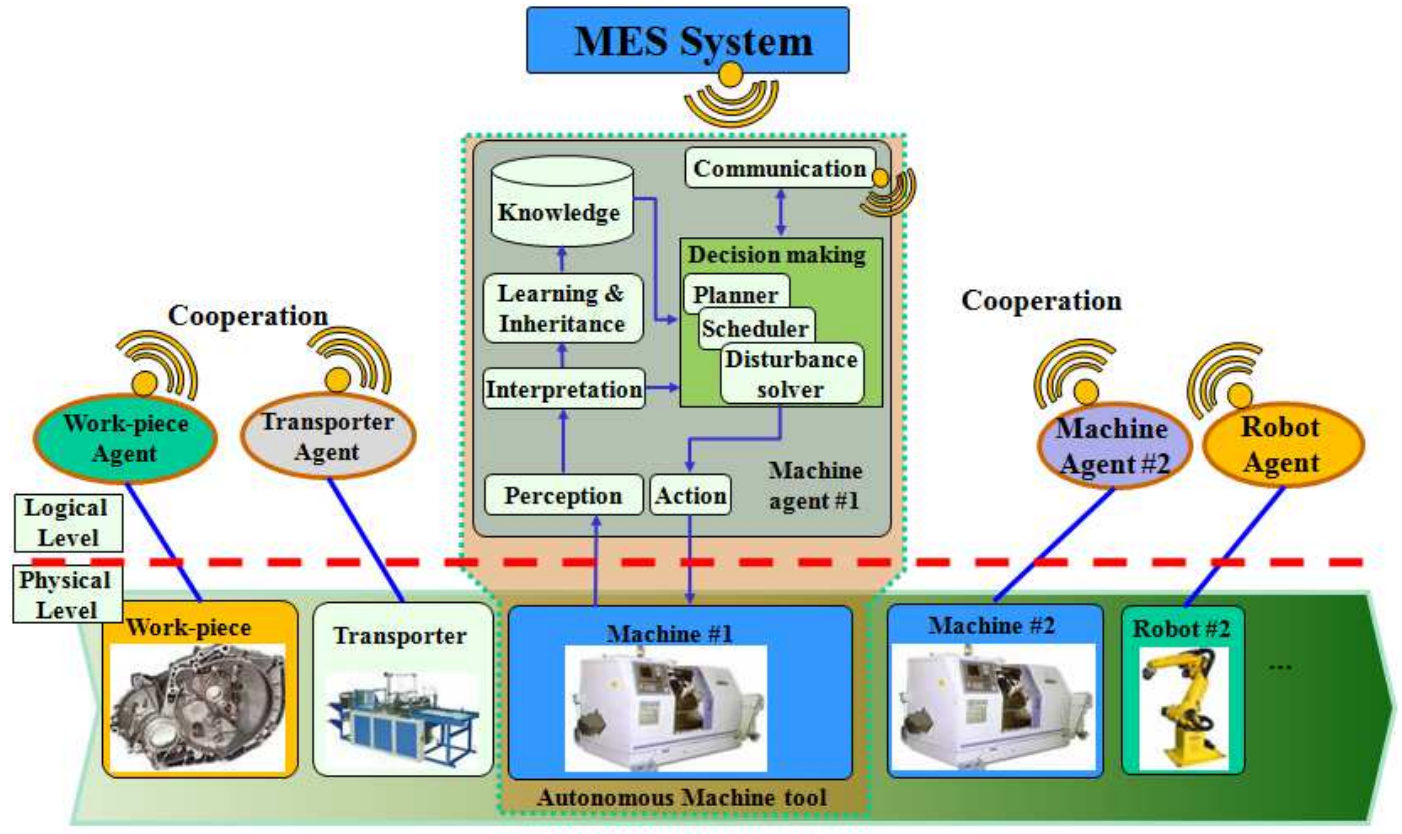

Figure 2. Autonomous machining system. 
In case the system does not have disturbances, the MES manages the machining process. In the case of disturbances such as tool wear, machine breakdown cognitive agents manage the machining process to ensure that machining processes are carried out continuously. For example, in the case of the tool wear, the machine adjusts the cutting conditions in consideration of the tool wear amount in the allowable range, and does not affect the quality of machining process. When a machine fails, the remaining machines will cooperate to find a suitable machine to do the work of the breakdown machine.

To connect the devices in the machining system with the application software needs to use communication protocols such as MTConnect, OPC (process control protocol for linking and embedding objects) $[20,21]$. In this study, the protocol for connecting between the CNC machine tools as well as other equipments in the system such as robots, workpiece, and transporter is OPC. The machines communicate with each others via the cognitive agents in the wireless environment using messages in XML (Extensible Markup Language) format.

\section{TECHNOLOGIES FOR REALIZING THE SYSTEM}

\subsection{Cognitive agent}
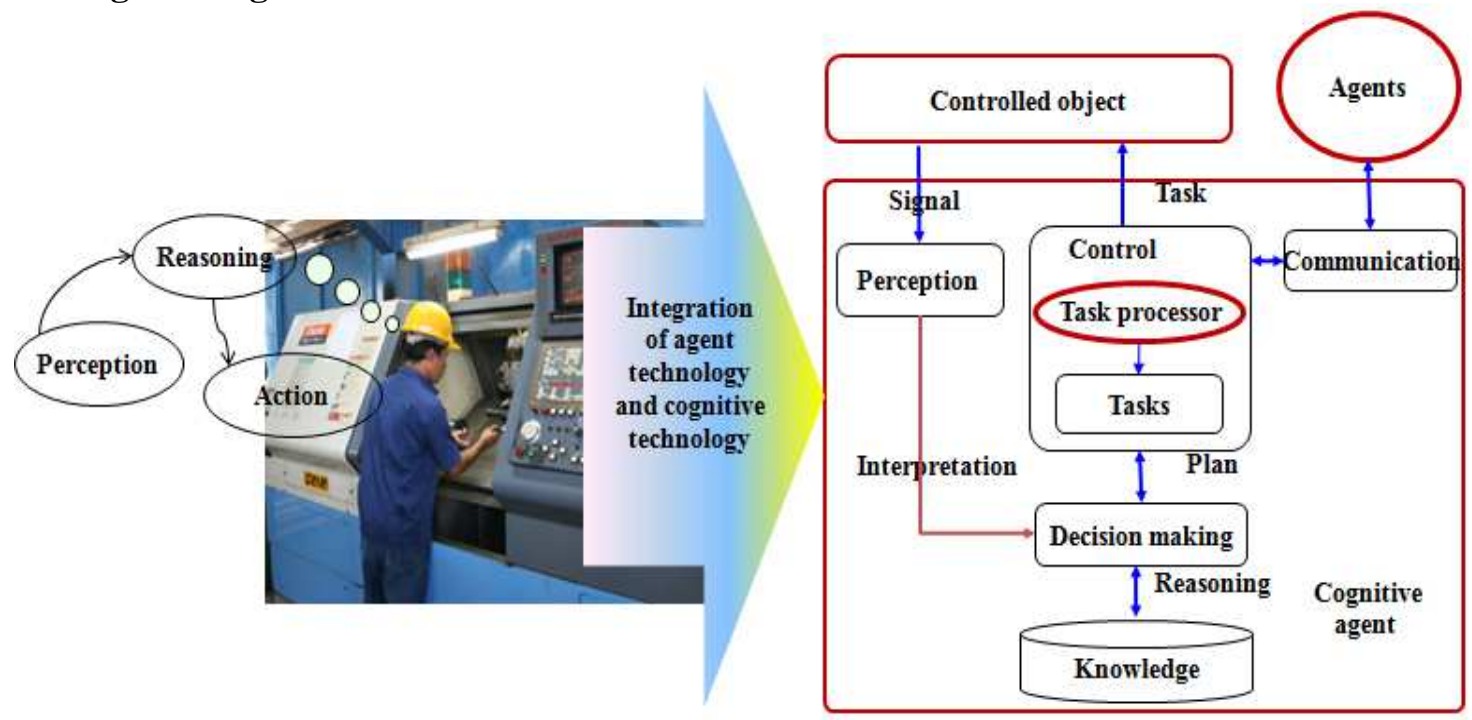

Figure 3.Architecture of a cognitive agent.

Cognitive agent $[18,19]$ is a computer program equipped with the artificial cognition to have the cognitive abilities of human. Cognitive activities are loop of three steps: perception, reasoning and action. According to the cognitive model of human for adapting to changes of environmental conditions, the architecture of cognitive agents has been proposed to apply in manufacturing field as shown in Figure 3. In which, the cognitive agent is made up of five basic modules: perception, decision making, knowledge, control, and communication. The perception module keeps the role for getting data of controlled objects such as machine tool, workpiece. The decision-making module is to drive out a responsible decision according to the obtained data and the knowledge of the cognitive agent. The control module processes the plan to tasks and activates the commands to the controlled object. The interaction among agents is done 
through the communication module. The knowledge module stores the plans and action mechanisms of the cognitive agent.

\subsection{Swarm intelligence}

In the nature, swarm intelligence is built from simple interactions of individuals and with their environment. Swarm intelligence is expressed through mechanisms to adapt to environmental change which shows in the study of biology, such as ant colony, fish school, birds, and the cooperation of human in problem solving.

Ant colony shows swarm intelligence as finding the shortest path from food source to their nests through simple interactions among them using a chemical substance called "pheromone" $[22,23]$. Ant colony adapts to environmental changes by changing the relationship between the members. Applying this principle to the manufacturing field in which the manufacturing system is considered as a combination of autonomous and collaborative entities, the manufacturing system adapts with disturbances by comparing the ability of the machine to the requirements of the product. Each machine has a value of "pheromone" to pass a particular type of disturbances, and the machine having the highest pheromone value is selected to perform the work of the breakdown machine.

\subsection{Information technology infrastructure}

Radio frequency identification (RFID) technology and sensor systems have an important role in changing the way to control, automatic manufacturing, and data acquisition, as well as in connection with information systems of the upper level such as ERP (enterprise resource planning), SCM (supply chain management), or CRM (customer relationship management) [24]. These technologies allow to reduce the costs, system downtime, and to increase productivity. RFID plays an important role in monitoring the process in real time, monitoring the objects of system such as workpiece, materials due to this technology allows to read and write data into the RFID tag attached directly to objects.

Wireless sensor network (WSN) includes temperature sensors, pressure sensors, force sensors, or vibration sensors. WSN is a tool for collecting data in real time. It plays an important role in monitoring the status of the machine which allows improving the quality and performance of the machine [25].

\section{PROGRAMMING THE SYSTEM}

Cognitive agents were programmed using .NET and C \#. The systematic structure of the machining system based on the cognitive agents is shown in Figure 4. There are three core issues to implement the cognitive agents, which are the communication protocol, agent behavior and database as well as information flow among the equipments in the system to perform the function. Agent interacts with MES and other agents through XML format.

OPC protocol is used for communication between the agents with PLC (programmable logic controllers). PLC is connected to the physical devices of the machining system such as the RFID reader, disturbance input, and alarm devices. The database includes process information, agent address for interacting in the network, the pheromone value, and disturbance database are built using SQL Server ${ }^{\mathrm{TM}}$ 2005. Agents use the "search" method for diagnosis and classification of disturbances. According to disturbance types, cognitive agent reasons to make a decision 
using the "adjust" or "collaboration" method. In cooperation, the agent uses the "calculate" function to generate the pheromone value. Then, the "negotiate" process is carried out among agents to find out the agent with the highest pheromone value. This agent transfers the machining plan of the breakdown machine to the selected machine tool. These functions of the agent are done using the database of process information; agent database in which SQL (structured query language) is used for queries.

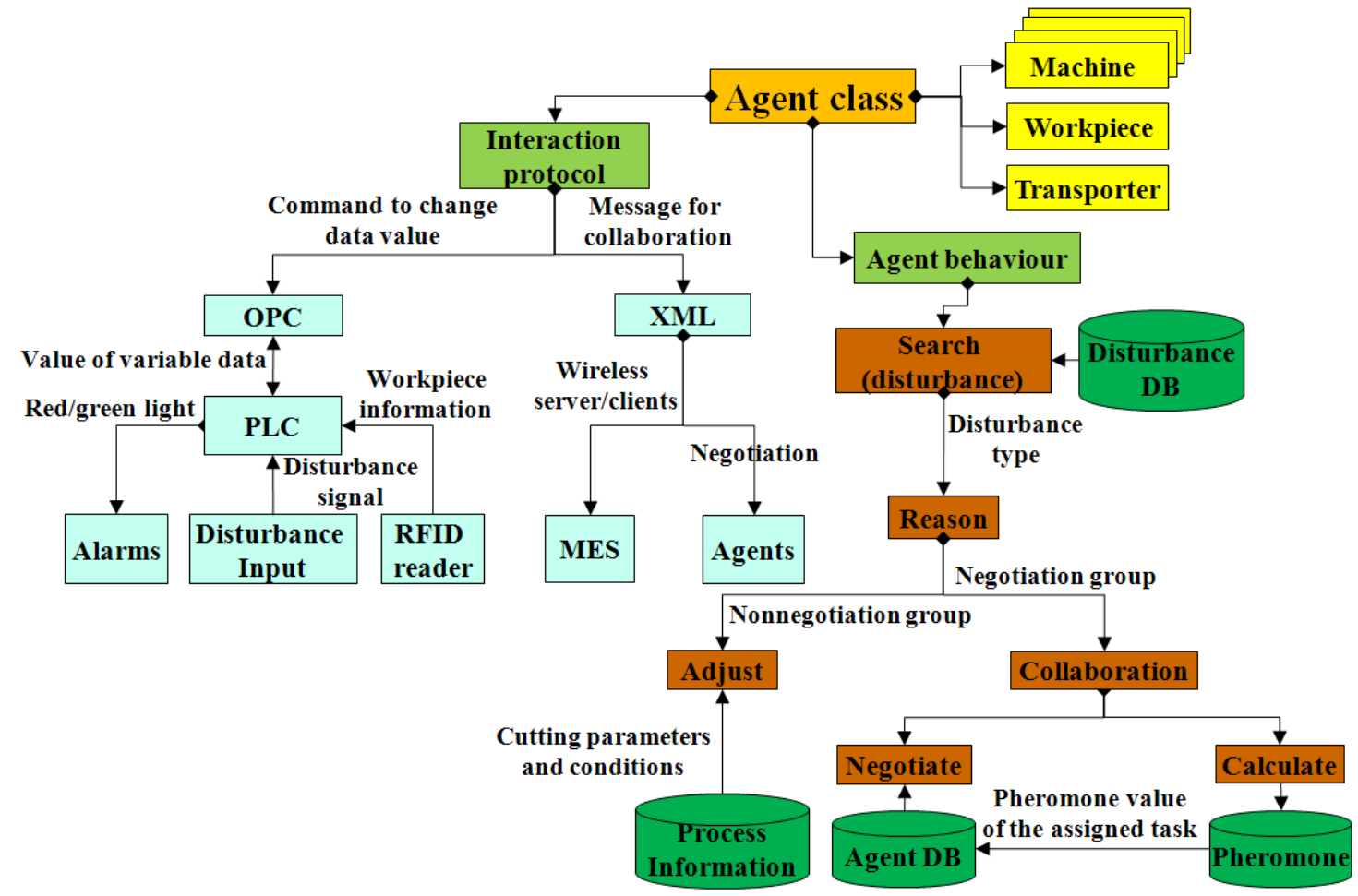

Figure 4. Structure of the cognitive agent based machining system.

\section{EVALUATION OF THE DEVELOPED SYSTEM}

\subsection{Hardware structure of the experimental model}

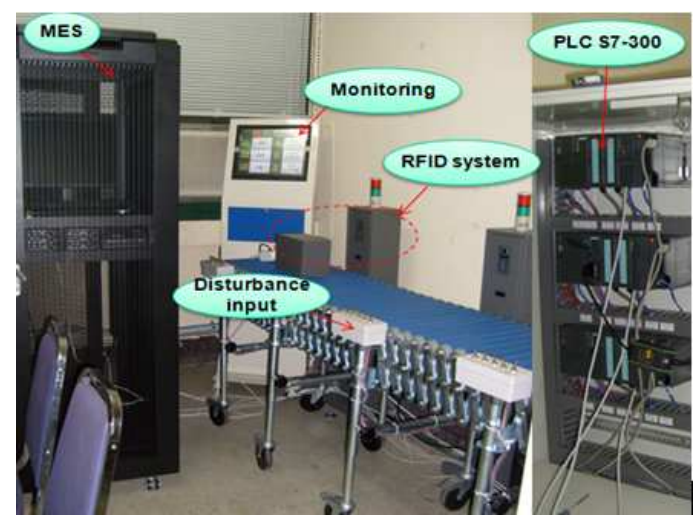

Figure 5. Hardware structure of the experimental model. 
Hardware structure of the experimental model is shown in Figure 5. In order to save investment costs, the disturbance-input device (device on/off) is used to generate the disturbance.

PLC considered as the controller of the machine tool receives the process information from the MES system and activates the machining operations. Process information of the system is displayed on the monitoring screen. Workpiece information is collected via RFID system. Each computer including three agents (machine agent, workpiece agent, and transport agent) is responsible for managing the machining workpiece on a machine tool.

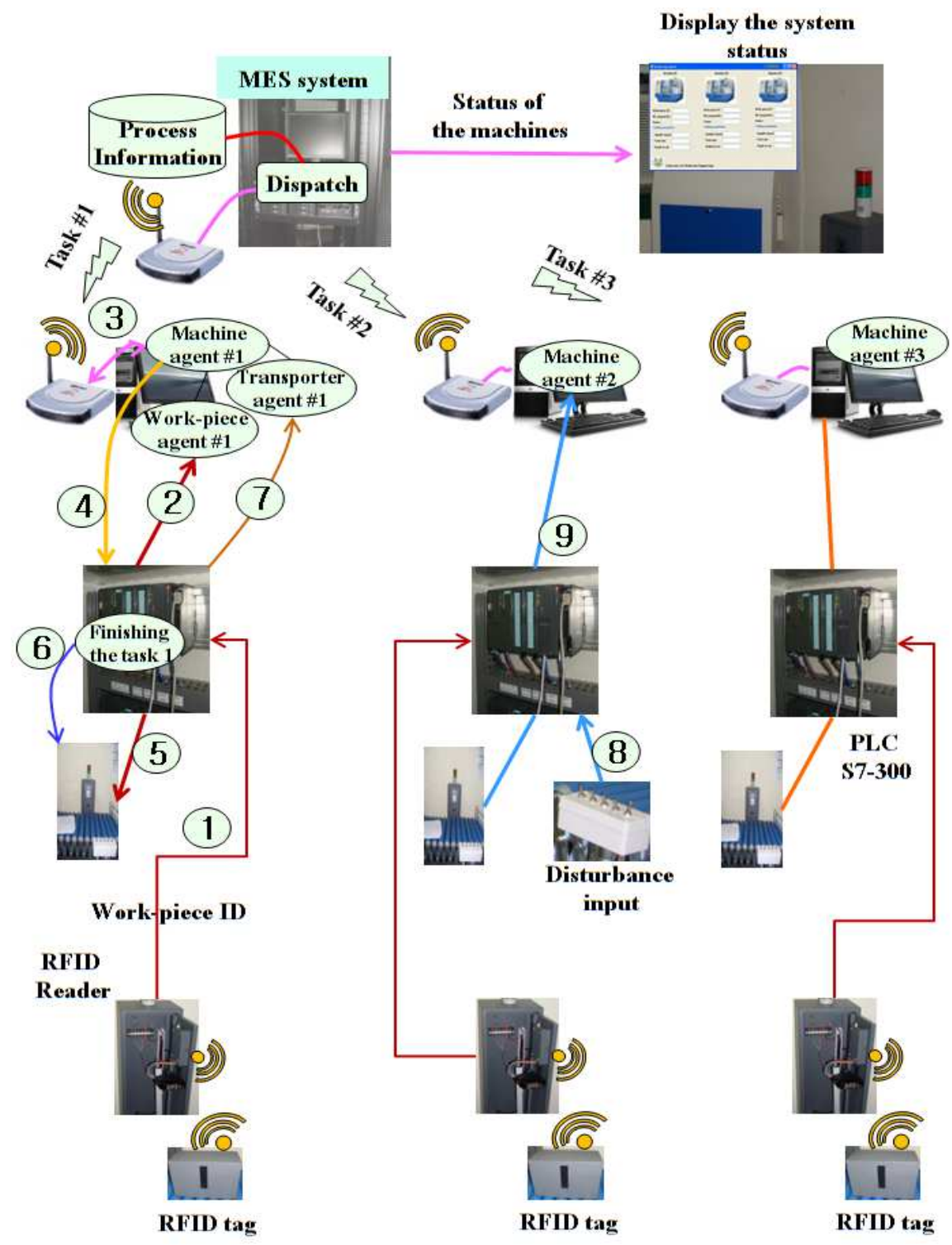

Figure 6. Working mechanism of the system.

Working principle of the system shown in Figure 6 is explained as follows:

- The RFID reader sends the workpiece information to PLC (described by 1 ). 
- Workpiece agent receives workpiece ID from the PLC and sent to the machine agent (described by 2 ).

- Machine agen requests task from MES (described by 3).

- Machine agent sends task to PLC (described by 4).

- PLC activates the green light (described by 5).

- After the operation finish, the green light is off (described by 6).

- PLC sends a signal to the transport agent to transfer the workpiece to the next machine (described by 7).

- Generating the disturbance (expressed by 8).

- $\quad$ PLC sends a signal to the machine agent (described by 9).

- Agent overcomes the disturbance by itself or in cooperation with other agents.

\subsection{Software structure of the experimental model}

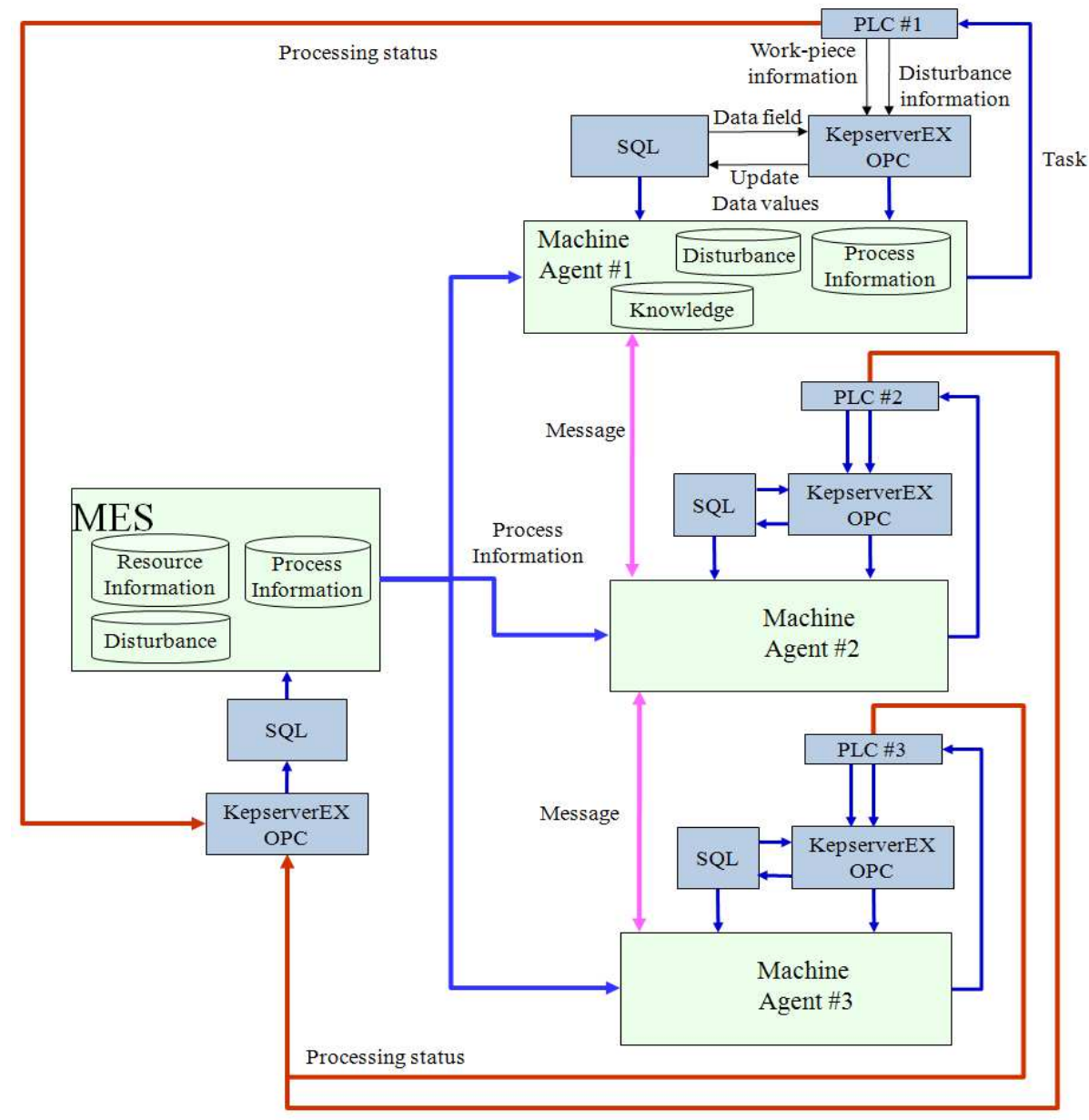

Figure 7. Software structure of the developed system. 
Figure 7 shows the software structure of the developed system. Agent communicates with MES and other agents through XML message which uses the 802.11 protocol for wireless communication. OPC protocol in KEPServerEx ${ }^{\mathrm{TM}}$ software is used for communication between agents with the PLC device. SQL is used to communicate with databases.

\subsection{Communication protocol}

Communication diagram among devices in the experimental model shown in Figure 8 is explained as follows:

- Communication protocol between the RFID tag attached on the workpiece and the RFID reader is UHF: ISO18000-6C (Gen2) standard for RFID Thingmagic ${ }^{\mathrm{TM}}$ devices.

- Communication protocol between the RFID reader and the PLC is wired with an RFID reader connected via USB and a side connected PLC S7-300 via RS 232.

- Disturbance-input devices connect to the PLC through DI port (digital input) of the PLC.

- Alarm light connects to the PLC by wire through CP 341-RS232C port of the PLC.

- PLC connects to computer by wire.

- Communication among computers and computers with MES via wireless devices which are Buffalo ${ }^{\mathrm{TM}}$ Ethernet converter.

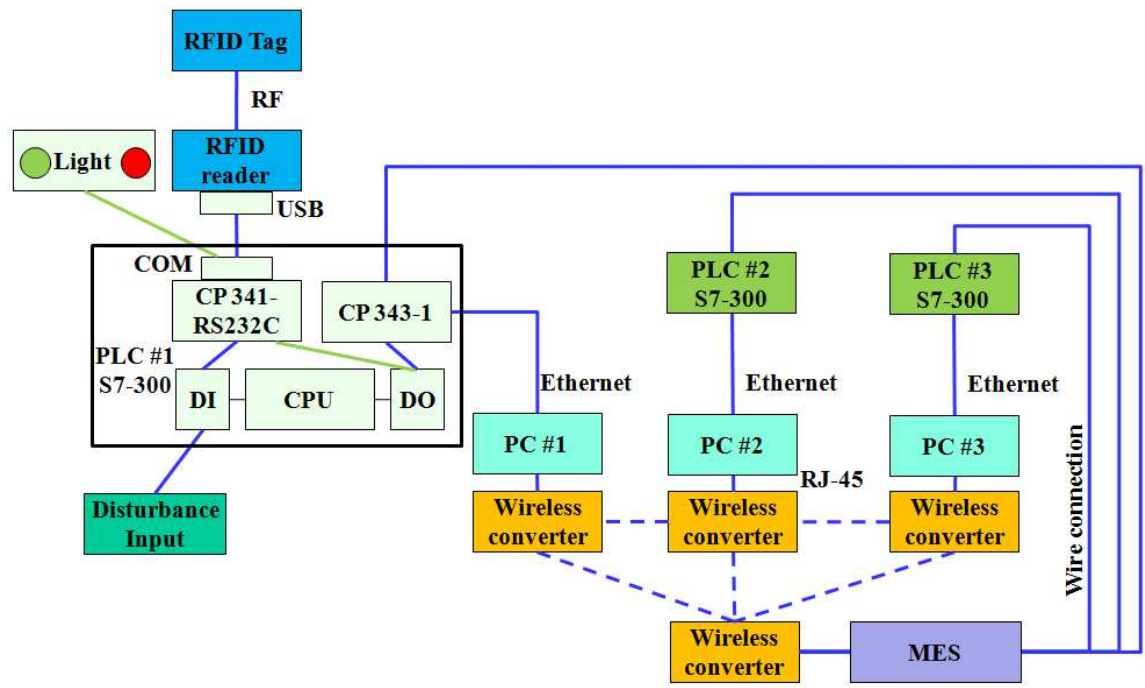

Figure 8.Comunication protocol among devices.

\subsection{Experimental results}

Figure 9 shows the self-adjusting mechanism of the agent about the cutting condition when machining on a lathe in the case of tool wear. Cognitive agents were programmed using .NET and C\#. Machine agent \#1 receives disturbance signals from the PLC \#1 through software KEPServerEx ${ }^{\mathrm{TM}}$ (described by 1 ). Then, the disturbance is diagnosed (described by 2 ). If the disturbance belongs to the non-negotiation type, for example tool wear (described by 3 ), the agent adjusts the cutting parameters. After changing the cutting parameters (described by 4), the cognitive agent send the new cutting parameters to the controller of machine tool. 


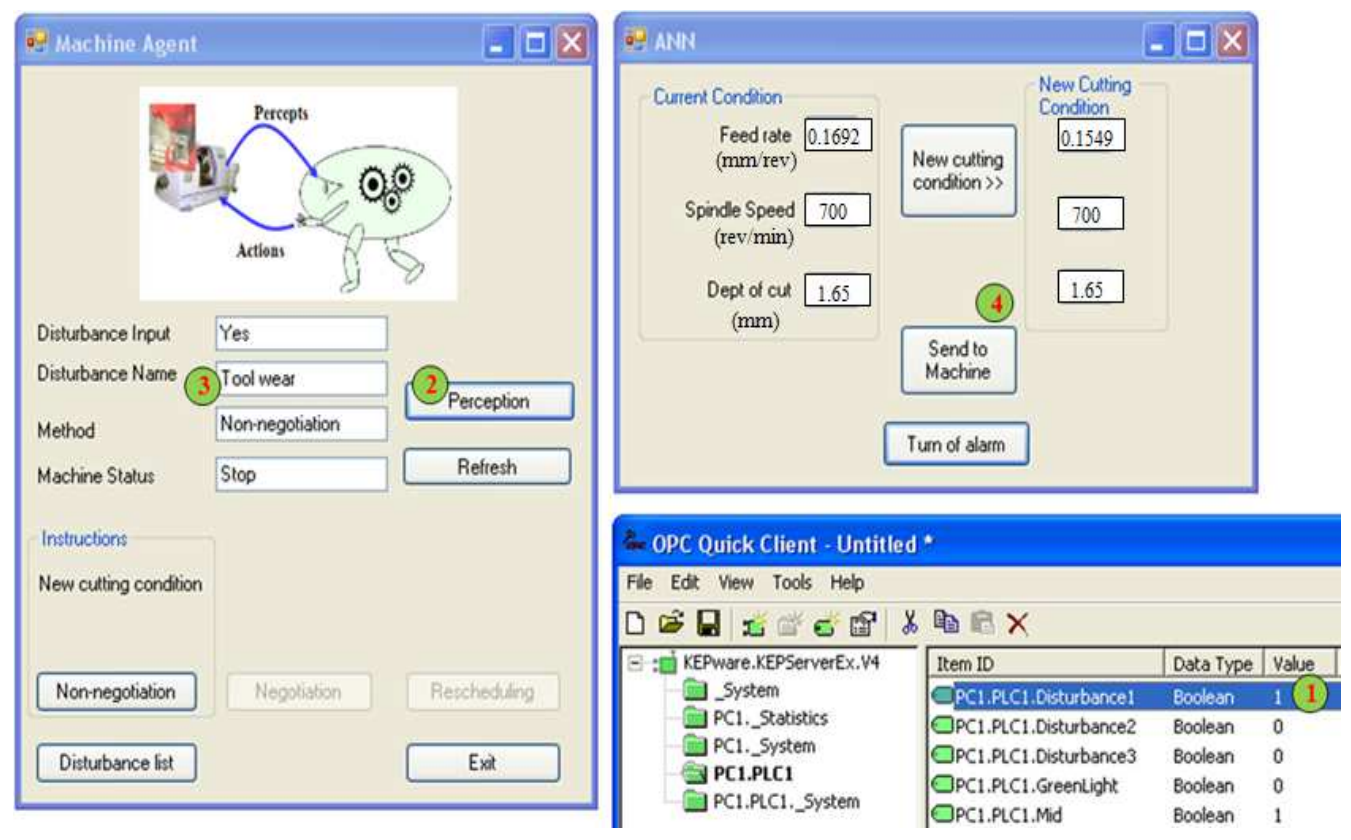

Figure 9. Screenshot of the system in the case of tool wear.

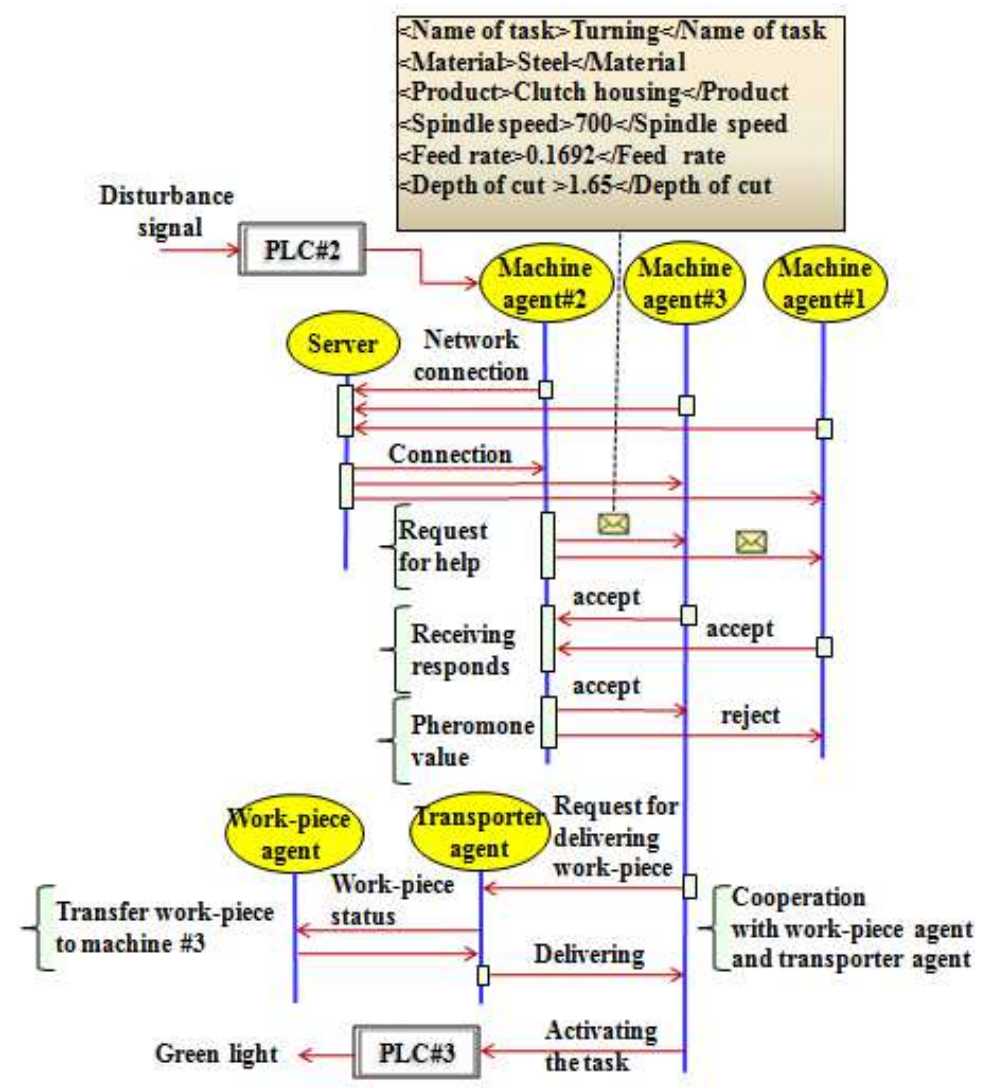

Figure 10. Agent negotiation. 


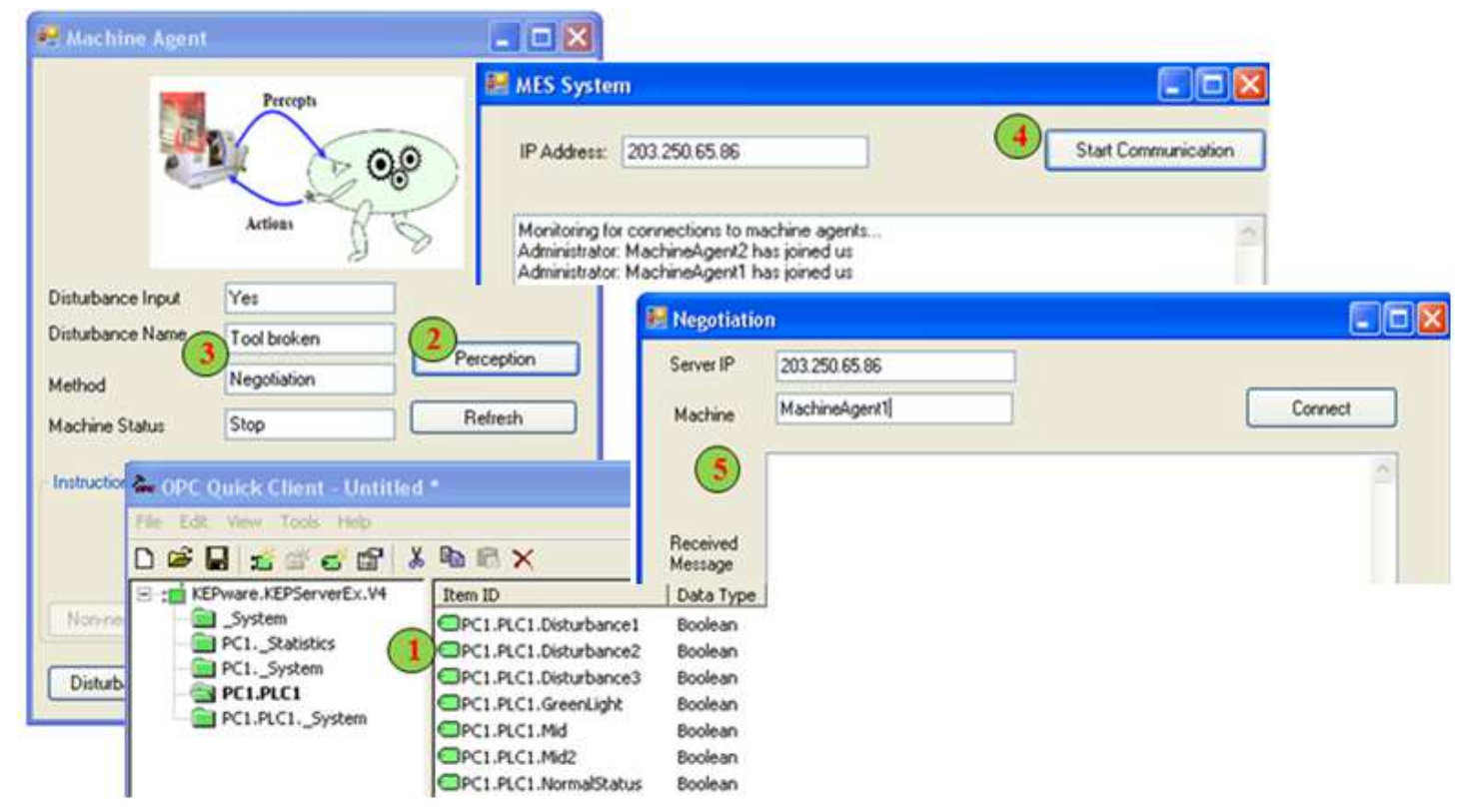

Figure 11. Screenshot of the machine agent in negotiation case.

The negotiation process of agents is shown in Figure 10. Machine agent \#2 sends requests for help to the remaining machine agents. Messages contain the process information, the address of the receiver. Agents negotiate to find an alternate route.

Screenshot of the machine agent shown in Figure 11 presents the response of the system in case of tool break. Machine agent \#2 receives the disturbance signal from the PLC \#2 via KEPServerEx ${ }^{\mathrm{TM}}$ software (described by 1 ). Then, the disturbance is diagnosed (described by 2 ). If the disturbance belongs to the negotiation type, for example broken tool (described by 3 ), the network server/client is established (described by 4). Agents cooperate to overcome the disturbances (described by 5 ).

\section{APPLICATION OF THE AUTONOMOUS MACHINING SYSTEM}

To applythe proposed AMS to the real machining system needs to synthesize, analyze the disturbance types happening in the system;to build laws for agent for making a decision. In this research, the AMS is applied to ensure the workpiece quality in the case of the tool wear using the self-adjusting mechanism of agent.

Figure 12 shows the integration of modules of machine agent to diagnose the tool wear amount when machining and generating a new cutting parameters corresponding to the tool wear amount to ensure the value of surface roughness of the machined product within allowed limits. Accordingly, the recorded signals from the sensors will be amplified and transferred to neural networks ANN \#1, in order to predict the tool wear. If the value of the surface roughness in consideration of tool wear exceeds the allowed limit, the system will report a notice to replace tool. Conversely, ANN \#2 is used to generate a new cutting condition. In this research, neural network ANN \#1 is used to model and predict the amount of tool wear in turning. Configuration of ANN \#1 is 6-10-1. The input layer includes 6 neurons: cutting speed $v$, feed rate $f$, cutting depth $a_{p}$, force, cutting time, the initial tool wear. The hidden layer includes 10 neurons. The 
output layer contains only one neuron that is the amount of tool wear. To get this structure, the number of learning is stopped after 2000 iterations; with the smallest deviation is 0.025 . Results of prediction are shown in Table 1.

In the machining process, if the amount of tool wear affects the machining quality $R_{a}$ then the new cutting parameters are generated using artificial neural network ANN \#2. ANN \#2 is structured 3-3-6-1 with two hidden layers. The input parameters were: cutting speed $v$, amount of tool wear $T_{w}$, cutting depth $a_{p}$. Output layer is composed of the feed rate parameter. The learning is stopped after 2000 iterations with the obtained smallest error 0.28. Results on the optimal amount of feed rate corresponding to the amount of tool wear are shown in Table 2.

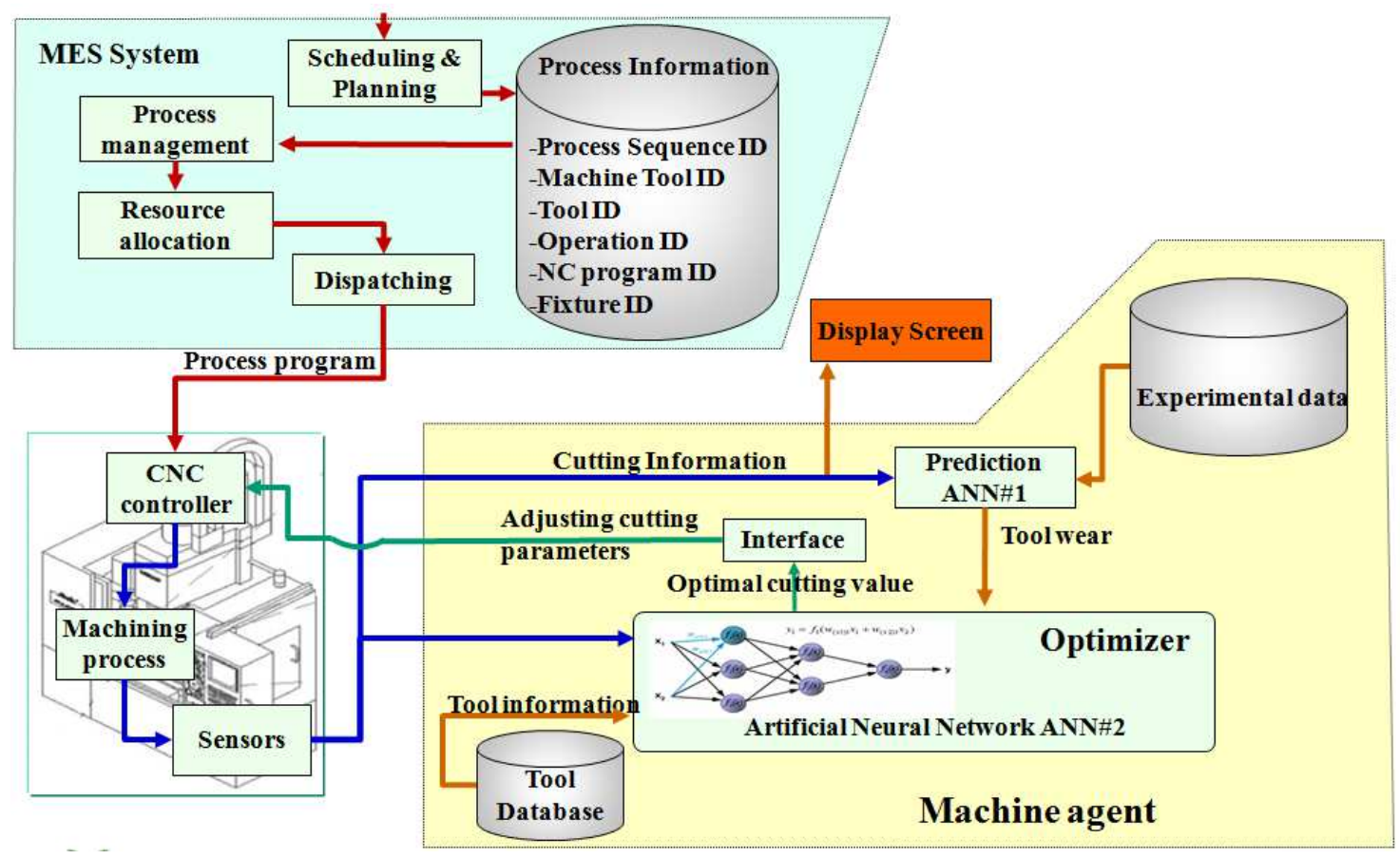

Figure 12. Prediction of the tool wear amount and generation of the new cutting parameters.

Table 1. Result of predicting the tool wear and comparison with the experimental data.

\begin{tabular}{cccccccccc}
\hline $\begin{array}{c}\text { Test Cutting } \\
\text { force } \\
(\mathrm{N})\end{array}$ & $\begin{array}{c}\text { Feed rate } \\
(\mathrm{mm} / \mathrm{rev})\end{array}$ & $\begin{array}{c}\text { Depth } \\
\text { of cut } \\
(\mathrm{mm})\end{array}$ & $\begin{array}{c}\text { Processing } \\
\text { time }(\mathrm{mm})\end{array}$ & $\begin{array}{c}\text { Cutting } \\
\text { speed } \\
(\mathrm{m} / \mathrm{min})\end{array}$ & $\begin{array}{c}\text { Initial } \\
\text { tool } \\
\text { wear } \\
(\mathrm{mm})\end{array}$ & $\begin{array}{c}\text { Actual } \\
\text { tool } \\
\text { wear } \\
(\mathrm{mm})\end{array}$ & $\begin{array}{c}\text { Predicted } \\
\text { tool wear } \\
(\mathrm{mm})\end{array}$ & $\begin{array}{c}\text { Error } \\
(\mathrm{mm})\end{array}$ \\
\hline 1 & 609 & 0.1449 & 1.5 & 5 & 160 & 0 & 0.140 & 0.133 & 0.007 \\
\hline 2 & 608 & 0.1398 & 1.5 & 10 & 160 & 0 & 0.174 & 0.173 & 0.001 \\
\hline 3 & 607 & 0.1347 & 1.5 & 20 & 160 & 0 & 0.208 & 0.205 & 0.003 \\
\hline 4 & 609 & 0.1449 & 1.5 & 30 & 160 & 0 & 0.292 & 0.279 & 0.013 \\
\hline 5 & 619 & 0.1680 & 2.0 & 5 & 160 & 0 & 0.165 & 0.147 & 0.015 \\
\hline 6 & 610 & 0.1500 & 2.0 & 20 & 160 & 0 & 0.195 & 0.216 & -0.021 \\
\hline
\end{tabular}


Table 2. Results of generation of the feed rate and comparison with the experimental data.

\begin{tabular}{ccccccc}
\hline Test & $\begin{array}{c}\text { Cutting } \\
\text { speed } \\
(\mathrm{m} / \mathrm{min})\end{array}$ & $\begin{array}{c}\text { Amount of tool } \\
\text { wear }(\mathrm{mm})\end{array}$ & $\begin{array}{c}\text { Depth of cut } \\
(\mathrm{mm})\end{array}$ & $\begin{array}{c}\text { Expected } \\
\text { feed rate } \\
(\mathrm{mm} / \mathrm{rev})\end{array}$ & $\begin{array}{c}\text { Generated } \\
\text { feed rate } \\
(\mathrm{mm} / \mathrm{rev})\end{array}$ & Error \\
\hline 1 & 160 & 0.158 & 1.635 & 0.29 & 0.270 & 0.020 \\
\hline 2 & 160 & 0.174 & 1.755 & 0.45 & 0.423 & 0.027 \\
\hline 3 & 160 & 0.180 & 1.800 & 0.55 & 0.500 & 0.050 \\
\hline 4 & 160 & 0.230 & 2.175 & 1.11 & 1.010 & 0.100 \\
\hline 5 & 160 & 0.290 & 2.626 & 1.61 & 1.580 & 0.030 \\
\hline 6 & 160 & 0.248 & 2.310 & 1.19 & 1.170 & 0.020 \\
\hline
\end{tabular}

\section{CONCLUSIONS}

Recommendationfor applying new technologies such as cognitive agent and swarm intelligence in manufacturing control field to replace the centralized control of the current manufacturing system to a distributed control by building an autonomous machining system to reduce system downtime in the case of disturbances is a new contribution of this research.

The experimental results show that the cognitive agent is capable to overcome disturbances by itselfas well as the cooperation ability with other agents. For applying the proposed autonomous machining system to the real machining system, further researches such as processing data from multiple sensor networks in the machining system as well as the laws for agents to drive out decisions with each specific disturbance must be carried out.

Acknowledgements. This research was funded by Vietnam National Foundation for Science and Technology Development (NAFOSTED) under grant number 107.01-2014.23.

\section{REFERENCES}

1. Christian Brecher - Integrative production technology for high-wage countries, SpringerVerlag Berlin Heidelberg 2012.

2. Tran Van Dich - Flexible manufacturing system (FMS) and Computer integrated manufacturing (CIM), Science and Technics Publishing House, 2001.

3. Zaeh M. F., Beetz M., Shea K., Reinhart G., Bender K., Lau C., Ostgathe M., Vogl W., Wiesbeck M., Engelhard M., Ertelt C., Rühr T., Friedrich M., Herle S - The cognitive factory, In: EIMaraghy HA (ed.) Changeable and reconfigurable manufacturing systems, Springer (2009) 355-371.

4. Phan Bui Khoi, Banh Tien Long - Multidisciplinary - linked effectiveness of science and technology in mechanical- automation and mechatronic engineering, Journal of Science and Technology 50 (6) (2012) 891-898.

5. Tran Ngoc Hien, Vo Hoai Son - A study of the influence of feed rate to roughness surface in turning using artificial neural networks, Proceedings of 4th National conference in mechanical engineering, Ho Chi Minh City, Vietnam, 11/2015.

6. Kristina S., Christoph E., Thomas G., Farhad A. - Design-to-fabrication automation for the cognitive machine shop, Advanced Engineering Informatics 24 (2010) 251-268. 
7. Tran Van Khiem - Research on usability of adaptive control techniques to improve the machining ability of CNC machine tool, Le Quy Don University of Science and Technology, PhD dissertation, 2011.

8. Thai Thi Thu Ha - Research, design and manufacture of intelligent and special numerical controllers $(\mathrm{CNC})$ for the complex process and system, Science and Technology Program in automation field KC.03.2005.

9. Park H. S., Tran N. H. - An intelligent manufacturing system with biological principles, International Journal of CAD/CAM 10 (2010) 39-50.

10. Wang L., Gao R. X. - Condition monitoring and control for intelligent manufacturing, Springer, 2006.

11. Nguyen Hong Thai - Kinematics of the multi-axis CNC milling machine type psk: Hexapod 3- axis CNC machine, Journal of Science and Technology 50 (4) (2012) 423439.

12. Nagurka M. L., Englert P. J. - Toward an intelligent machine tool for flexible manufacturing, Robotics and Computer - Integrated Manufacturing 6 (1989) 229-236.

13. Sutherland J. W., Ferreira P. M., DeVor R. E., Kapoor S. G. - An integrated approach to machine tool system analysis, design and control, The 3rd International Conference on Computer - Aided Production Engineering (1988) 429-445.

14. Sato T., Kakino Y., Saraie H. - Proposal of the extended system framework of intelligent machine tool, Japan-USA Symposium on Flexible Automation, 2002.

15. Koren Y. - Adaptive control systems for machining, Manuf Rev 2 (1989) 6-15.

16. Tarng Y. S., Wang Y. S. - A new adaptive controller for constant turning force, J AdvManufTechnol 9 (1994) 211-216.

17. Shirase K., Nakamoto K., Arai E., Moriwaki T. - Digital copy milling- autonomous milling process control without an NC program. Robot ComputIntegrManuf21 (2005) 312-317.

18. Le Tan Hung, Tu Minh Phuong, Huynh Quyet Thang - Agents - Agent oriented software engineering, Science and Technics Publishing House, 2006.

19. Park H. S., Tran N. H. - A cognitive agent based manufacturing system for adapting to disturbances, International Journal of Control, Automation, and Systems 10 (2012) 806816.

20. Richard Zurawski - Industrial communication technology handbook, CRC Press Taylor \& Francis Group, 2015.

21. Wilamowski B. M., Irwin J. D. - Industrial communication systems, CRC Press Taylor \& Francis Group, 2011.

22. Garg A., Gill P., Rathi P., Amardeep, Garg K. K. - An insight into swarm intelligence, International Journal of Recent Trends in Engineering 2 (2009) 42-44.

23. Peeters P., Brussel H. V., Valckenaers P., Wyns J., Bongaerts L., Kollingbaum M., Heikkila T. - Pheromone based emergent shop floor control system for flexible flow shops, Artificial Intelligence in Engineering 15 (2001) 343-352.

24. Oliver P. Günther, Kletti W., Kubach U. - RFID in Manufacturing, Springer, 2008.

25. Katsumi T., Kenji K. - Ubiquitous sensor network system, NEC Technical Journal 1 (1) (2006) 78-82. 